\title{
STATISTICS OF CLUSTERS IN BINARY LINEAR LATTICES
}

\author{
B. U. FELDERHOF \\ Instituut voor Theoretische Fysica der Rijksuniversiteit, \\ Utrecht, Nederland
}

Received 20 August 1971

\section{Synopsis}

The statistics of clusters in binary linear lattices is studied on the assumption that the relative weight of an $A_{l}$ or $B_{m}$ cluster is determined only by its size $l$ or $m$, and is independent of the location of the cluster on the chain. The average cluster numbers and the variance of their fluctuations are calculated for two probability ensembles, the quasi-grand ensemble where the total number of units $N$ varies, and the canonical ensemble where $N$ is fixed. It is shown that in the latter case in the limit of large $N$ the statistics of clusters is fully described by the cluster densities $\rho_{l}$ and $\rho_{m}$, and a correlation function $g_{\mathrm{AB}}(R)$ for which a susceptibility theorem is derived.

1. Introduction. We consider linear systems of $N$ basic units which can be in either of two states, A or B. A configuration of the system is represented by a sequence of $N$ symbols $\mathrm{A}$ and $\mathrm{B}$ in some order, e.g.

\section{AAA BB AABA ... BAAAB.}

Of the numerous examples of such systems we mention linear arrays of $N$ spins $\frac{1}{2}$, the linear lattice gas of $N$ sites, polymer chains with adsorption along the chain, copolymers consisting of two types of units, polypeptides with the letters $A$ and $B$ specifying whether a monomer unit is in the helix or coil state, and polynucleotides with A signifying bonding of units on opposite strands (helix) and B implying the absence of a bond (coil).

A particular configuration will be denoted by $\boldsymbol{s} \equiv\left(s_{1}, s_{2}, \ldots, s_{N}\right)$, where the variables $s_{i}$ take the values A or B. Each configuration $s$ is also completely specified by a linear sequence of alternating $\mathrm{A}$ clusters and $\mathrm{B}$ clusters, e.g.

$$
\mathrm{A}_{3} \mathrm{~B}_{2} \mathrm{~A}_{2} \mathrm{~B}_{1} \ldots \mathrm{A}_{3} \mathrm{~B}_{1} \text {. }
$$

In equilibrium statistical mechanics each configuration is assigned a probability $P(s)$ on the basis of the physical properties of the chain. We consider a class of systems for which the statistical weight of a configuration is determined solely by the numbers of $A_{l}$ clusters and $B_{m}$ clusters present, 
independent of the location of the clusters along the chain. Hence $P(s)$ is the same for all $s$ with the same set of occupation numbers $\left\{n_{\mathrm{A}_{t}}, n_{\mathrm{B}_{m}}\right\}$. It is not implied that the clusters do not interact but only that the interactions of any cluster with its surroundings are independent of the nature of the surroundings so that their effect may be incorporated in the statistical weight of the given cluster. Since we are mainly interested in the statistical properties for large $N$, we neglect the fact that frequently the end clusters have different statistical weights. If desired the end effects may be treated separately.

A detailed account of the statistics of such binary linear systems with particular application to the helix-coil transition in biopolymers is presented in the monograph by Poland and Scheraga ${ }^{1}$ ). Of the earlier work we mention papers by $\mathrm{Hill}^{2}$ ), Zimm and Bragg ${ }^{3}$ ), I,ifson and co-workers ${ }^{4-7}$ ), and Gibbs and DiMarzio ${ }^{8}$ ). In developing a theory of the kinetics of binary linear systems we have found it desirable to extend the existing theories in some respects. In the present paper we investigate in detail the averages of the occupation numbers $\left\{n_{\mathrm{A}_{t}}, n_{\mathrm{B}_{m}}\right\}$ and their fluctuations in various probability ensembles. We have chosen a coherent presentation and therefore must of necessity repeat some known results.

In section 2 we define distribution functions and calculate the corresponding partition functions. Section 3 is devoted to the study of cluster populations. In section 4 we investigate the behaviour in the limit of long chains, $N \rightarrow \infty$. In section 5 we apply some of our results to the case of the Ising chain. The paper is concluded with a discussion.

2. Distributions and partition functions. In this section we discuss probability distributions for the cluster occupation numbers $\left\{n_{\mathbf{A}_{\imath}}, n_{\mathbf{B}_{m}}\right\}$ and related partition functions. When no confusion can arise we shall abbreviate $n_{\mathrm{A}_{l}} \equiv n_{l}$ and $n_{\mathrm{B}_{m}} \equiv n_{m}$. A set of occupation numbers $\left\{n_{l}, n_{m}\right\}$ will be called a state of the system and will be denoted by $x \equiv\left\{n_{l}, n_{m}\right\}$. Furthermore we define

$$
\begin{array}{ll}
N_{\mathrm{A}}=\sum_{l=1}^{\infty} l n_{l}, & N_{\mathbf{B}}=\sum_{m=1}^{\infty} m n_{m}, \\
M_{\mathbf{A}}=\sum_{l=1}^{\infty} n_{l}, & M_{\mathrm{B}}=\sum_{m=1}^{\infty} n_{m} .
\end{array}
$$

Note that $N_{\mathrm{A}}+N_{\mathrm{B}}=N$ and that $M_{\mathrm{A}}$ and $M_{\mathrm{B}}$ can differ at most by unity. The smaller of $M_{\mathrm{A}}$ and $M_{\mathrm{B}}$ will be denoted by $M$. The number of configurations $s$ corresponding to a state $\boldsymbol{x}$ is given by

$$
\Omega(\boldsymbol{x})=\frac{M_{\mathbf{A}}(\boldsymbol{x}) !}{\prod_{l} n_{l} !} \frac{M_{\mathbf{B}}(\boldsymbol{x}) !}{\prod_{m} n_{m} !}\left(1+\delta_{M_{\mathbf{A}}, M_{\mathbf{B}}}\right),
$$

and will be called the volume of the state $\boldsymbol{x}$. If the number of units is pre- 
cisely $N^{\prime}$ it is convenient to introduce the conditional volume

$$
\Omega\left(\boldsymbol{x} ; N^{\prime}\right)=\Omega(\boldsymbol{x}) \delta\left[N(\boldsymbol{x})-N^{\prime}\right],
$$

where $\delta\left(N-N^{\prime}\right)=\delta_{N, N^{\prime}}$ is a Kronecker delta. If in an $N$ chain an $A_{l}$ cluster has weight $u_{l}$ and a $B_{m}$ cluster has weight $v_{m}$ the total weight of the state $x$ is

$$
G(\boldsymbol{x})=\prod_{l, m} u_{l}^{n_{l} v_{m}^{n_{m}}}
$$

Hence the probability distribution over states is given by

$$
P(\boldsymbol{x} ; N)=\Omega(\boldsymbol{x} ; N) G(\boldsymbol{x}) / Z_{N}
$$

where $Z_{N}$ is the canonical partition function

$$
Z_{N}=\sum_{x} \Omega(x ; N) G(x)
$$

In some cases, e.g. in polymerization equilibrium, one must also consider a distribution over $N$ with relative weight $z^{N}$. The corresponding quasi-grand distribution function ${ }^{5,6}$ ) is

$$
P(\boldsymbol{x} ; z)=z^{N} \Omega(\boldsymbol{x} ; N) G(\boldsymbol{x}) / \boldsymbol{\Xi}(z),
$$

where

$$
\Xi(z)=\sum_{N=0}^{\infty} z^{N} Z_{N}
$$

is the quasi-grand partition function. The first term in (2.8) is unity by convention. In contrast to the usual canonical and grand canonical ensembles in statistical mechanics the distributions (2.5) and (2.7) can give widely different results for the averages of observables, because the factor $\left(N !^{-1}\right.$ is missing in (2.7) and hence the distribution over $N$ is very broad.

In order to evaluate the partition functions it is convenient to consider constrained ensembles where the nature of the first and last cluster, and the total number of clusters are specified. Thus we introduce

$$
P^{\text {if }}(\boldsymbol{x} ; N, M)=\Omega^{\text {if }}(\boldsymbol{x} ; N, M) G(\boldsymbol{x}) / Z_{N, M}^{\text {if }},
$$

where $i$ and $f$ take the values $A$ or $B$ and indicate whether the first and last cluster are $\mathrm{A}$ or $\mathrm{B}$, and where

$$
\begin{aligned}
& \Omega^{\mathrm{if}}(\boldsymbol{x} ; N, M)=\Omega^{\mathrm{if}}(\boldsymbol{x} ; N) \delta[M(\boldsymbol{x})-M], \\
& Z_{N . M}^{\mathrm{if}}=\sum_{\boldsymbol{x}} \Omega^{\mathrm{if}}(\boldsymbol{x} ; N, M) G(\boldsymbol{x}) .
\end{aligned}
$$

The corresponding quasi-grand partition functions are

$$
\Xi_{M}^{\mathrm{if}}(z)=\sum_{N=0}^{\infty} z^{N} Z_{N, M}^{\mathrm{if}},
$$


where we use the convention

$$
Z_{0,0}^{\mathrm{AA}}=Z_{0,0}^{\mathrm{BB}}=0, \quad Z_{0,0}^{\mathrm{AB}}=Z_{0,0}^{\mathrm{BA}}=1 .
$$

Hence

$$
\Xi(z)=-1+\sum_{M=0}^{\infty}\left[\Xi_{M}^{\mathrm{AA}}(z)+\Xi_{M}^{\mathrm{AB}}(z)+\Xi_{M}^{\mathrm{BA}}(z)+\Xi_{M}^{\mathrm{BB}}(z)\right] .
$$

The partition functions (2.11) are easily evaluated in terms of the cluster generating functions

$$
U(z)=\sum_{l=1}^{\infty} z^{l} \mathfrak{u}_{l}, \quad V(z)=\sum_{m=1}^{\infty} z^{m y_{m}},
$$

and one finds explicitly

$$
\begin{aligned}
& \Xi_{M}^{\mathrm{AA}}(z)=U(z)[U(z) V(z)]^{M}, \\
& \Xi_{M}^{\mathrm{AB}}(z)=\Xi_{M}^{\mathrm{BA}}(z)=[U(z) V(z)]^{M}, \\
& \Xi_{M}^{\mathrm{BB}}(z)=V(z)[U(z) V(z)]^{M},
\end{aligned}
$$

so that

$$
\boldsymbol{\Xi}(z)=[1+U(z)+V(z)+U(z) V(z)] /[1-U(z) V(z)] .
$$

The partition functions $Z_{N}^{\text {if }}$ are found from

$$
Z_{N}^{\mathrm{if}}=\frac{1}{2 \pi \mathrm{i}} \oint \frac{\Xi^{\mathrm{if}}(z)}{z^{N+1}} \mathrm{~d} z,
$$

where the integration is along a contour encircling the origin of the complex $z$ plane. The free energy per unit in the thermodynamic limit $N \rightarrow \infty$ is determined by the singularity $z_{0}$ of $\Xi^{\text {if }}(z)$ nearest to the origin. It is given by

$$
\varphi=-k T \lim _{N \rightarrow \infty} N^{-1} \ln Z_{N}^{\text {if }}=k T \ln z_{0},
$$

where $k$ is Boltzmann's constant and $T$ absolute temperature. The free energy $\varphi$ is independent of the end conditions. A phase transition in the sense of a non-analyticity in the free energy can occur 9,10 ) because the singularity $z_{0}$ can arise in two ways, either as a root of the equation

$$
U(z) V(z)=1 \quad \text { (exterior condition), }
$$

or alternatively because one of the cluster generating functions $U(z)$ or $V(z)$ fails to converge. For definiteness we may choose $U(z)$ as the series with smallest radius of convergence. Then a phase transition can occur as one varies the physical parameters because of a switchover from the exterior condition (2.19) to

$$
z=\lim _{l \rightarrow \infty}\left[u_{l}\right]^{-1 / l} \quad \text { (interior condition). }
$$


When the latter condition prevails the statistical properties of the system in the thermodynamic limit are dominated by the A cluster of infinite size. Since we are interested in nontrivial cluster populations we shall henceforth assume that the free energy is determined by a (simple) root of eq. (2.19). Under this condition we find

$$
\begin{aligned}
& Z_{N}^{\mathrm{AA}}=U\left(z_{0}\right) \rho z_{0}^{-N}\left[1+\mathcal{O}\left(\lambda^{-N}\right)\right], \\
& Z_{N}^{\mathrm{AB}}=Z_{N}^{\mathrm{BA}}=\rho z_{0}^{-N}\left[1+\mathcal{O}\left(\lambda^{-N}\right)\right], \\
& Z_{N}^{\mathrm{BB}}=V\left(z_{0}\right) \rho z_{0}^{-N}\left[1+\mathcal{O}\left(\lambda^{-N}\right)\right],
\end{aligned}
$$

with

$$
\lambda=\left(z_{1} / z_{0}\right)(1+\varepsilon),
$$

where $\varepsilon$ is small and positive and where $z_{1}$ is the root of (2.19) with nextsmallest absolute value. Finally $\rho$ in $(2.21)$ is given by

$$
\rho^{-1}=\left[z \frac{\mathrm{d} \ln U(z)}{\mathrm{d} z}+z \frac{\mathrm{d} \ln V(z)}{\mathrm{d} z}\right]_{z=z_{0}},
$$

where we have used $U\left(z_{0}\right) V\left(z_{0}\right)=1$.

The calculation of statistical averages of observables $f(s)$ dependent on the configurations $s$ may be performed in a similar fashion. We define

$$
F(x)=\sum_{s} f(s) \delta[x(s)-x] / \Omega(x),
$$

where the delta function specifies that the state $\left\{n_{l}(\boldsymbol{s}), n_{m}(\boldsymbol{s})\right\}$ corresponding to the configuration $\boldsymbol{s}$ must be $\boldsymbol{x}$. The quasi-grand average for specified end clusters is given by

$$
\langle f(s)\rangle_{z}^{\text {if }}=\langle F(x)\rangle_{z}^{\text {if }}=\sum_{x} F(x) P^{\text {if }}(x ; z),
$$

and similarly one defines $\langle f(s)\rangle_{N}^{\text {if }}$ as an average over the canonical probability distribution (2.5) for given end terms. The canonical and quasigrand averages are related by

$$
Z_{N}^{\mathrm{if}}\langle F(x)\rangle_{N}^{\mathrm{if}}=\frac{1}{2 \pi \mathrm{i}} \oint \frac{\boldsymbol{\Xi}^{\mathrm{if}}(z)\langle F(x)\rangle_{z}^{\mathrm{if}}}{z^{N+1}} \mathrm{~d} z .
$$

The quasi-grand average is easily evaluated, and hence the canonical average is calculated using (2.26).

3. Cluster populations. In this section we calculate the average cluster numbers and their fluctuations in both the quasi-grand and canonical ensemble. Owing to the broad distribution over $N$ the fluctuations in cluster numbers in the quasi-grand ensemble are enormous. The expressions are slightly dependent on end effects and take their most elegant form for 
$\mathrm{i}=\mathrm{A}, \mathrm{f}=\mathrm{B}$, or vice versa. One easily finds

$$
\begin{aligned}
& \left\langle n_{l}\right\rangle_{z}^{\mathrm{AB}}=u_{l} \frac{\partial}{\partial u_{l}} \ln \Xi^{\mathrm{AB}}(z)=\frac{z^{l} u_{l}}{U(z)}\left[\Xi^{\mathrm{AB}}(z)-1\right], \\
& \left\langle n_{m}\right\rangle_{z}^{\mathrm{AB}}=v_{m} \frac{\partial}{\partial v_{m}} \ln \Xi^{\mathrm{AB}}(z)=\frac{z^{m_{v_{m}}}}{V(z)}\left[\Xi^{\mathrm{AB}}(z)-1\right] .
\end{aligned}
$$

Hence

$$
\begin{aligned}
& \langle M\rangle_{z}^{\mathrm{AB}}=\Xi^{\mathrm{AB}}(z)-1, \\
& \langle N\rangle_{z}^{\mathrm{AB}}=\left[\langle l\rangle_{z}+\langle m\rangle_{z}\right]\langle M\rangle_{z}^{\mathrm{AB}},
\end{aligned}
$$

where $\left\langle l_{z}\right.$ and $\langle m\rangle_{z}$ are internal cluster moments defined generally by

$$
\begin{aligned}
& \langle l k\rangle_{z}=\sum_{l=1}^{\infty} l k z l u_{l} / U(z), \\
& \left\langle m^{k}\right\rangle_{z}=\sum_{m=1}^{\infty} m^{k} z^{m} v_{m} / V(z) .
\end{aligned}
$$

From (2.23) and (3.2) one finds

$$
\rho_{z}=\langle M\rangle_{z}^{\mathrm{AB}} /\langle N\rangle_{z}^{\mathrm{AB}},
$$

so that $\rho_{z}$ may be interpreted as the average A- or B-cluster density per unit. It is simply related to the degree of association $\chi_{z}$ by

$$
\rho_{z}=\left[\langle l\rangle_{z}+\langle m\rangle_{z}\right]^{-1}=1 / 2 \chi_{z}
$$

Of equal importance is the fraction of $\mathrm{A}$ units $\theta_{z}$ which is given by

$$
\theta_{z}=\left\langle N_{\mathrm{A}}\right\rangle_{z}^{\mathbf{\Lambda} \mathrm{B}} /\langle N\rangle_{z}^{\mathbf{A} \mathrm{B}}=\langle l\rangle_{z} \mid\left[\langle l\rangle_{z}+\langle m\rangle_{z}\right] .
$$

As $z$ tends to $z_{0}$ the averages of both $M$ and $N$ diverge but their ratio tends to a finite value. In order to indicate averages in the thermodynamic limit $z \rightarrow z_{0}$ we shall simply omit the subscript $z$. The fraction $\theta_{z}$ tends to a value $\theta$ between 0 and 1 , and $\rho_{z}$ tends to a value $\rho$ between 0 and $\frac{1}{2}$ as $z \rightarrow z_{0}$.

For the variance of the fluctuations in the cluster numbers one finds

$$
\begin{aligned}
& \left\langle n_{l} n_{l^{\prime}}\right\rangle_{z}^{\mathbf{A B}}-\left\langle n_{l}\right\rangle_{z}^{\mathbf{A B}}\left\langle n_{l^{\prime}}\right\rangle_{z}^{\mathbf{A B}}=\left\langle n_{l}\right\rangle_{z}^{\mathbf{A B}} \delta_{l l^{\prime}}+\left\langle n_{l}\right\rangle_{z}^{\mathbf{A B}}\left\langle n_{l^{\prime}}\right\rangle_{z}^{\mathbf{A B}}, \\
& \left\langle n_{l} n_{m}\right\rangle_{z}^{\mathbf{A B}}-\left\langle n_{l}\right\rangle_{z}^{\mathbf{A B}}\left\langle n_{m}\right\rangle_{z}^{\mathbf{A B}}=\left\langle n_{l}\right\rangle_{z}^{\mathbf{A B}}\left\langle n_{m}\right\rangle_{z}^{\mathbf{A B}}\left(1+1 /\langle M\rangle_{z}^{\mathbf{A B}}\right), \\
& \left\langle n_{m} n_{m^{\prime}}\right\rangle_{z}^{\mathbf{A B}}-\left\langle n_{m}\right\rangle_{z}^{\mathbf{A B}}\left\langle n_{m^{\prime}}\right\rangle_{z}^{\mathbf{A B}}=\left\langle n_{m}\right\rangle_{z}^{\mathbf{A B}} \delta_{m m^{\prime}}+\left\langle n_{m}\right\rangle_{z}^{\mathbf{A} \mathbf{B}}\left\langle n_{m^{\prime}}\right\rangle_{z}^{\mathbf{A B}} .
\end{aligned}
$$

Consequently

$$
\begin{aligned}
& \left\langle M^{2}\right\rangle_{z}^{\mathrm{AB}}-\left(\langle M\rangle_{z}^{\mathrm{AB}}\right)^{2}=\langle M\rangle_{z}^{\mathrm{AB}}\left(1+\langle M\rangle_{z}^{\mathrm{AB}}\right), \\
& \left\langle N^{2}\right\rangle_{z}^{\mathbf{A B}}-\left(\langle N\rangle_{z}^{\mathbf{A B}}\right)^{2}=\left(\langle N\rangle_{z}^{\mathbf{A B}}\right)^{2}+\left\langle(l+m)^{2}\right\rangle_{z}\langle M\rangle_{z}^{\mathrm{AB}} .
\end{aligned}
$$


Therefore there are large fluctuations in the cluster numbers and in the total number of units owing to the wide distribution over $N$ in the quasigrand ensemble.

The canonical averages are expressed most convenicntly in terms of the cluster weights $\left\{u_{l}, v_{m}\right\}$ and the canonical partition function. Thus one finds from (2.26)

$$
\begin{aligned}
& \left\langle n_{l}\right\rangle_{N}^{\mathrm{if}}=\sum_{N^{\prime}, N^{\prime \prime}} Z_{N^{\prime}}^{\mathrm{iB}} u_{l} Z_{N^{\prime \prime}}^{\mathrm{Bf}} \delta\left(N^{\prime}+l+N^{\prime \prime}-N\right) / Z_{N}^{\mathrm{if}}, \\
& \left\langle n_{m}\right\rangle_{N}^{\mathrm{if}}=\sum_{N^{\prime}, N^{\prime \prime}}^{\sum} Z_{N^{\prime}}^{\mathrm{iA}} v_{m} Z_{N^{\prime \prime}}^{\mathrm{Af}} \delta\left(N^{\prime}+m+N^{\prime \prime}-N\right) / Z_{N}^{\mathrm{if}},
\end{aligned}
$$

where we use the convention (2.12). Hence we find the simple relation ${ }^{4}$ )

$$
\left\langle n_{l}\right\rangle_{N}^{\text {if }}=\frac{u_{l} Z_{N-l+1}^{\text {if }}}{u_{1} Z_{N}^{\text {if }}}\left\langle n_{\mathrm{A}_{1}}\right\rangle_{N-l+1}^{\text {if }},
$$

and similarly for $\left\langle n_{m}\right\rangle_{N}^{\text {if }}$. From (3.9) we find for the averages of the total number of $\mathrm{A}$ and $\mathrm{B}$ clusters

$$
\begin{aligned}
& \left\langle M_{\mathrm{A}}\right\rangle_{N}^{\text {if }}=-\delta_{\mathrm{Bf}}+\sum_{N^{\prime}} Z_{N^{\prime}}^{\mathrm{iB}} Z_{N-N^{\prime}}^{\mathrm{Af}} / Z_{N}^{\mathrm{if}}, \\
& \left\langle M_{\mathrm{B}}\right\rangle_{N}^{\mathrm{if}}=-\delta_{\mathrm{Af}}+\sum_{N^{\prime}} Z_{N^{\prime}}^{\mathrm{iA}} Z_{N-N^{\prime}}^{\mathrm{Bff}} / Z_{N}^{\mathrm{if}},
\end{aligned}
$$

where we have used

$$
\begin{aligned}
& Z_{N}^{\mathrm{Af}}=\sum_{l} u_{l} Z_{N-l}^{\mathrm{Bf}}+\delta_{N, 0} \delta_{\mathrm{Bf}} \\
& Z_{N}^{\mathrm{Bf}}=\sum_{m} v_{m} Z_{N-m}^{\mathrm{Af}}+\delta_{N, 0} \delta_{\mathrm{Af}} .
\end{aligned}
$$

In order to evaluate the second moments of the cluster numbers we note from (2.26) that

$$
\left\langle n_{l} n_{l^{\prime}}\right\rangle_{N}^{\mathrm{if}}=\left\langle n_{l}\right\rangle_{N}^{\mathrm{if}} \delta_{l l^{\prime}}+\sum_{R>0}\left[\left\langle n\left(l|R| l^{\prime}\right)\right\rangle_{N}^{\mathrm{if}}+\left\langle n\left(l^{\prime}|R| l\right)\right\rangle_{N}^{\mathrm{if}}\right],
$$

where $n\left(l|R| l^{\prime}\right)$ is the number of pairs of $\mathrm{A}_{l}$ and $\mathrm{A}_{l^{\prime}}$ clusters separated by $R$ units; the notation $\left(l|R| l^{\prime}\right)$ indicates the order along the chain. The average number of such pairs is

$$
\begin{aligned}
& \left\langle n\left(l|R| l^{\prime}\right)\right\rangle_{N}^{\mathrm{if}} \\
& \quad=\sum_{N^{\prime}, N^{\prime \prime}} Z_{N^{\mathrm{i}}}^{\mathrm{iB}} u_{l} Z_{R}^{\mathrm{BB}} u_{l^{\prime}} Z_{N^{m}}^{\mathrm{Bf}} \delta\left(N^{\prime}+l+R+l^{\prime}+N^{\prime \prime}-N\right) / Z_{N}^{\mathrm{if}} .
\end{aligned}
$$

Similarly one finds

$$
\left\langle n_{l} n_{m}\right\rangle_{N}^{\mathrm{if}}=\sum_{R \geqslant 0}\left[\langle n(l|R| m)\rangle_{N}^{\mathrm{if}}+\langle n(m|R| l)\rangle_{N}^{\mathrm{if}}\right],
$$


with

$$
\begin{aligned}
& \langle n(l|R| m)\rangle_{N}^{\text {if }} \\
& \quad=\sum_{N^{\prime}, N^{\prime \prime}} Z_{N^{\prime}}^{\mathrm{iB}} u_{l} Z_{R}^{\mathrm{BA}} v_{m} Z_{N^{\prime \prime}}^{\mathrm{Af}} \delta\left(N^{\prime}+l+R+m+N^{\prime \prime}-N\right) / Z_{N^{\circ}}^{\text {if }}
\end{aligned}
$$

The expression for $\left\langle n_{m} n_{m^{\prime}}\right\rangle_{N}^{\text {if }}$ is found from (3.13) by a change of indices.

4. Thermodynamic limit. The canonical averages derived in the last section will now be studied in the thermodynamic limit $N \rightarrow \infty$. With the aid of (2.21) one finds from (3.9)

$$
\begin{aligned}
& \rho_{l}=\lim _{N \rightarrow \infty} \frac{1}{N}\left\langle n_{l}\right\rangle_{N}^{\text {if }}=\rho z_{0}^{l} u_{l} / U\left(z_{0}\right), \\
& \rho_{m}=\lim _{N \rightarrow \infty} \frac{1}{N}\left\langle n_{m}\right\rangle_{N}^{\text {if }}=\rho z_{0}^{m_{\gamma_{m}} / V\left(z_{0}\right),}
\end{aligned}
$$

irrespective of the end conditions. Hence we may write

$$
\rho_{l}=\rho f_{l}, \quad \rho_{m}=\rho f_{m},
$$

where the distributions $f_{l}$ and $f_{m}$ are normalized to unity. Note that the densities $\rho_{l}$ and $\rho_{m}$ are identical with those found from the quasi-grand averages in the limit $z \rightarrow z_{0}, e . g$.,

$$
\rho_{l}=\lim _{z \rightarrow z_{0}}\left\langle n_{l}\right\rangle_{z}^{\text {if }} /\langle N\rangle_{z}^{\text {if }} \text {. }
$$

In the same manner one finds

$$
\begin{aligned}
& \rho\left(l|R| l^{\prime}\right)=\lim _{N \rightarrow \infty} N^{-1}\left\langle n\left(l|R| l^{\prime}\right)\right\rangle_{N}^{\text {if }}=\rho_{l} \rho_{l^{\prime}} g_{\mathrm{AA}}(R) \\
& \rho(l|R| m)=\rho(m|R| l)=\rho_{l} \rho_{m} g_{\mathrm{AB}}(R) \\
& \rho\left(m|R| m^{\prime}\right)=\rho_{m} \rho_{m^{\prime}} g_{\mathrm{BB}}(R)
\end{aligned}
$$

where

$$
\begin{aligned}
& g_{\mathrm{AA}}(R)=\left[\rho V\left(z_{0}\right)\right]^{-1} z_{0}^{R} Z_{R}^{\mathrm{BB}}, \\
& g_{\mathrm{AB}}(R)=\rho^{-1} z_{0}^{R} Z_{R}^{\mathrm{AB}}, \\
& g_{\mathrm{BB}}(R)=\left[\rho U\left(z_{0}\right)\right]^{-1} z_{0}^{R} Z_{R}^{\mathrm{AA}} .
\end{aligned}
$$

From (2.21) and (4.2) it follows that at large distances $(R \rightarrow \infty)$ these correlation functions tend to unity exponentially, though not necessarily monotonically. From (3.12) we have the simple relationships

$$
\begin{aligned}
& g_{\mathrm{AA}}(R)=\sum_{m} f_{m} g_{\mathrm{AB}}(R-m), \\
& g_{\mathrm{BB}}(R)=\sum_{l} f_{l} g_{\mathrm{AB}}(R-l),
\end{aligned}
$$




$$
\begin{array}{r}
g_{\mathrm{AB}}(R)=\rho^{-1} \delta(R)+\sum_{l} f_{l} g_{\mathrm{AA}}(R-l) \\
=\rho^{-1} \delta(R)+\sum_{m} f_{m} g_{\mathrm{BB}}(R-m) .
\end{array}
$$

From (3.15) and (4.6) we find

$$
\rho_{l, m} \equiv \rho(l|0| m)=\rho^{-1} \rho_{l \rho_{m}} .
$$

These results are easily generalized. Thus one has in obvious notation

$$
\rho\left(l_{1}\left|R_{1}\right| m_{1}\left|R_{2}\right| \ldots \mid l_{n}\right)=\prod_{j=1}^{n} \rho_{l}, \prod_{k=1}^{n-1} \rho_{m_{k}} \prod_{i=1}^{2 n-2} g_{\mathrm{AB}}\left(R_{i}\right) .
$$

In particular, since $g_{\mathrm{AB}}(0)=\rho^{-1}$, the density of $k$ neighbouring clusters is just the product of the individual cluster densities multiplied by a factor $\rho^{1-k}$. The statistics of clusters along the chain in the thermodynamic limit $N \rightarrow \infty$ is completely characterized by the cluster densities $\rho_{l}$ and $\rho_{m}$, and the correlation function $g_{\mathrm{AB}}(R)$.

In order to evaluate the fluctuations in cluster numbers in the canonical ensemble in the limit $N \rightarrow \infty$ the above calculation must be carried one step further in the asymptotic expansion with respect to $N$. The final results for the fluctuations are independent of the nature of the clusters at the ends of the chain. In order to simplify the notation of intermediate steps in the calculation which do depend on the end effects we choose for definiteness the symmetric case (i, f) $=(A, B)$. From (3.9) and (4.5) we have in the limit of large $N$

$$
\begin{gathered}
\left.\left\langle n_{l}\right\rangle\right\rangle_{N}^{\mathrm{AB}}-\rho_{l} \sum_{N^{\prime}} g_{\mathrm{AB}}\left(N^{\prime}\right) g_{\mathrm{AA}}\left(N-N^{\prime}-l\right) / g_{\mathrm{AB}}(N) \\
=\rho_{l}\left[N-l-1+\sigma_{\mathrm{AB}}+\sigma_{\mathrm{AA}}\right]+\mathcal{O}(1),
\end{gathered}
$$

where $N-l-1$ is the number of possible locations of an $\mathrm{A}_{l}$ cluster on the chain and where

$$
\sigma_{\alpha \beta}=\sum_{R=0}^{\infty}\left[g_{\alpha \beta}(R)-1\right]+\delta_{\alpha \beta}, \quad \alpha, \beta=\mathrm{A} \text { or } \mathrm{B} .
$$

Similarly one finds from (3.14)

$$
\begin{aligned}
\sum_{n}\langle n & \left.\left(l|R| l^{\prime}\right)\right\rangle_{N}^{\mathrm{AB}} \\
& =\rho l \rho l^{\prime} \sum_{N^{\prime}, R} g_{\mathrm{AB}}\left(N^{\prime}\right) g_{\mathrm{AA}}(R) g_{\mathrm{AA}}\left(N-N^{\prime}-l-R-l^{\prime}\right) / g_{\mathrm{AB}}(N) \\
& -\rho l \rho l^{\prime}\left[\frac{1}{2} N^{2}-\left(l+l^{\prime}+\frac{3}{2}\right) N\right. \\
& \left.+\frac{1}{2}\left(l+l^{\prime}+1\right)\left(l+l^{\prime}+2\right)+N\left(\sigma_{\mathrm{AB}}+2 \sigma_{\mathrm{AA}}\right)\right]+\mathcal{O}(1),
\end{aligned}
$$

where again the sum of the first three terms in square brackets is the number of possible locations of an ordered pair of $A_{l}$ and $A_{l^{\prime}}$ clusters. In 
the same fashion one finds

$$
\begin{aligned}
& \sum_{R}\langle n(l|R| m)\rangle\rangle_{N}^{\mathrm{AB}}=\rho_{l} \rho_{m}\left[\frac{1}{2} N^{2}-\left(l+m-\frac{1}{2}\right) N\right. \\
&\left.\quad+\frac{1}{2}(l+m-1)(l+m)+3 N \sigma_{\mathrm{AB}}\right]+\mathcal{O}(1), \\
& \sum_{R}\langle n(m|R| l)\rangle_{N}^{\mathrm{AB}}=\rho_{l} \rho_{m}\left[\frac{1}{2} N^{2}-\left(l+m+\frac{3}{2}\right) N\right. \\
&\left.\quad+\frac{1}{2}(l+m+1)(l+m+2)+N\left(\sigma_{\mathrm{AA}}+\sigma_{\mathrm{AB}}+\sigma_{\mathrm{BB}}\right)\right]+\mathcal{O}(1) .
\end{aligned}
$$

The additional results which are needed are easily found from the ones given above by a change of indices.

For the variances of the fluctuations in cluster numbers we finally find using (3.13) and (3.15)

$$
\begin{aligned}
\lim _{N \rightarrow \infty} & \frac{1}{N}\left[\left\langle n_{l} n_{l^{\prime}}\right\rangle_{N}^{\text {if }}-\left\langle n_{l}\right\rangle_{N}^{\text {if }}\left\langle n_{l^{\prime}}\right\rangle_{N}^{\text {if }}\right] \\
= & \rho_{l} \delta_{l l^{\prime}}+\rho_{l} \rho_{l^{\prime}}\left[2 \sigma_{\mathrm{AA}}-l-l^{\prime}-1\right], \\
\lim _{N \rightarrow \infty} & \frac{1}{N}\left[\left\langle n_{l} n_{m}\right\rangle_{N}^{\text {if }}-\left\langle n_{l}\right\rangle_{N}^{\text {if }}\left\langle n_{m}\right\rangle_{N}^{\text {if }}\right] \\
& =\rho_{l} \rho_{m}\left[2 \sigma_{\mathrm{AB}}-l-m+1\right], \\
\lim _{N \rightarrow \infty} & \frac{1}{N}\left[\left\langle n_{m} n_{m^{\prime}}\right\rangle_{N}^{\text {if }}-\left\langle n_{m}\right\rangle_{N}^{\text {if }}\left\langle n_{m^{\prime}}\right\rangle_{N}^{\text {if }}\right] \\
\quad= & \rho_{m} \delta_{m m^{\prime}}+\rho_{m} \rho_{m}\left[2 \sigma_{\mathrm{BB}}-m-m^{\prime}-1\right] .
\end{aligned}
$$

We stress again that these results are independent of the end clusters. The quantities on the right-hand sides are related by some identities which follow from (4.6),

$$
\begin{aligned}
& \rho \sigma_{\mathrm{AA}}=\rho \sigma_{\mathrm{AB}}+\rho+\theta-1, \\
& \rho \sigma_{\mathrm{BB}}=\rho \sigma_{\mathrm{AB}}+\rho-\theta,
\end{aligned}
$$

where we have used

$$
\langle l\rangle-\theta / \rho, \quad\langle m\rangle=(1-\theta) / \rho .
$$

Summing any of the three expressions (4.13) over the cluster indices one finds, using (4.14) and (4.15),

$$
\lim _{N \rightarrow \infty} \frac{1}{N}\left[\left\langle M_{\alpha} M_{\beta}\right\rangle_{N}^{\text {if }}-\left\langle M_{\alpha}\right\rangle_{N}^{\text {if }}\left\langle M_{\beta}\right\rangle_{N}^{\text {if }}\right]=\rho^{2}-\rho+2 \rho^{2} \sigma_{\mathrm{AB}}
$$

where $\alpha, \beta=A$ or $B$, so that the fluctuations in the total numbers of clusters $M_{\mathrm{A}}$ and $M_{\mathrm{B}}$ are independent of the indices $\mathrm{A}$ and $\mathrm{B}$, as they should since $M_{\mathrm{A}}$ and $M_{\mathrm{B}}$ can differ at most by unity. The expression on the right-hand side in $(4.16)$ is simply related to the fluctuations in size 
of individual clusters. This relation is found by noting that in the canonical ensemble the total number of units $N$ cannot fluctuate, so that

$$
\left\langle N^{2}\right\rangle_{N}^{\text {if }}=\left(\langle N\rangle_{N}^{\text {if }}\right)^{2}
$$

Hence one finds from (4.13)

$$
\rho^{2}-\rho+2 \rho^{2} \sigma_{\mathrm{AB}}=\rho^{3}\left[\left\langle l^{2}\right\rangle-\langle l\rangle^{2}+\left\langle m^{2}\right\rangle-\langle m\rangle^{2}\right] .
$$

A similar relationship between the fluctuations in the total number of clusters and the fluctuations in size of individual clusters was found in the continuum cluster-interaction model ${ }^{11}$ ).

The variance on the left side in (4.16) may be expressed in terms of a thermodynamic derivative and this means that the identity (4.16) has the nature of a susceptibility or compressibility theorem. In order to make the connection explicit it is expedient to introduce a thermodynamic variable conjugate to the total number of clusters. For example, each of the cluster weights $u_{l}$ may be multiplied by a factor $\zeta$, signalling the presence of an A cluster. The average number of A clusters in the canonical ensemble is then given by

$$
\left\langle M_{\mathrm{A}}\right\rangle_{N}^{\text {if }}=\zeta \frac{\partial}{\partial \zeta} \ln Z_{N}^{\mathrm{if}}(\zeta),
$$

or in the thermodynamic limit, using (2.21)

$$
\lim _{N \rightarrow \infty} \frac{1}{N}\left\langle M_{\mathrm{A}}\right\rangle_{N}^{\text {if }}=\rho=-\frac{\partial\left(\ln z_{0}\right)}{\partial(\ln \zeta)} .
$$

Similarly the variance in (4.16) is given by the second logarithmic derivative with respect to $\zeta$ and hence one finds the susceptibility theorem

$$
\rho+\rho^{2} \sum_{R=-\infty}^{\infty}\left[g_{\mathrm{AB}}(R)-1\right]=\zeta \frac{\partial \rho}{\partial \zeta},
$$

where by definition $g_{\mathrm{AB}}(--R)=g_{\mathrm{AB}}(R)$ and we have used $g_{\mathrm{AB}}(0)=\rho^{-1}$. In turn one may derive (4.17) from (4.20) using (2.23). These relations are of particular interest near a phase transition where the fluctuations can become very large and the susceptibility $\partial \rho / \partial \zeta$ may diverge.

5. Application to the Ising chain. The theory developed in the previous sections will now be applied to the particular case of the Ising chain. In this case the probability distribution $P(s ; N)$ in the canonical ensemble is given by

$$
P(s ; N)=\exp [-\beta E(s ; N)] / Z_{N},
$$

where $\beta=1 / k T$ and $E(s ; N)$ is the energy of a configuratiun of $N$ spins. We associate the state A with spin up, $s=+1$, and the state B with spin 
down, $s=-1$. For the Ising chain one assumes nearest neighbour interactions between spins and a magnetic field acting on each spin. In addition we assign an extra energy to the spins at the ends of the chain in order to give the end clusters the same weight as the interior clusters. Thus the energy is given by

$$
E(s ; N)=-J \sum_{j=1}^{N-1} s_{j} s_{j+1}-H \sum_{j=1}^{N} s_{j}-J\left(s_{1}+s_{N}-1\right),
$$

where $J$ is the bond energy and $H$ the magnetic field in suitablc units. It is easily seen that the energy of all configurations $s$ corresponding to the same state $x$ is the same and is given by

$$
E(x)=\sum_{l} n_{l} \varepsilon_{l}+\sum_{m} n_{m} \varepsilon_{m}
$$

with

$$
\varepsilon_{l}=-l(J+H), \quad \varepsilon_{m}=-m(J-H)+4 J,
$$

where $n_{l}$ is the number of clusters of $l$ spins up and $n_{m}$ the number of clusters of $m$ spins down. Hence the statistical weight of an $l$ cluster is

$$
\begin{array}{ll}
u_{l}=u^{l}, & u=\exp [\beta(J+H)], \\
v_{m}=u^{-z_{w}-2,} & w=\exp [\beta(J-H)] .
\end{array}
$$

The cluster generating functions are

$$
U(z)=u z /(1-u z), \quad V(z)=z /\left[u^{2} w(1-w z)\right] .
$$

The exterior condition (2.19) has two roots

$$
z_{0,1}=\mathrm{e}^{-\beta J} /\left\{\cosh \beta H \pm\left[\sinh ^{2} \beta H+\exp (-4 \beta J)\right]^{\hat{2}}\right\},
$$

and the explicit expressions for the canonical partition functions read

$$
\begin{aligned}
& Z_{N}^{\mathrm{AA}}=U\left(z_{0}\right) \rho_{0} z_{0}^{-N}+U\left(z_{1}\right) \rho_{1} z_{1}^{-N}, \\
& Z_{N}^{\mathrm{AB}}=\rho_{0} z_{0}^{-N}+\rho_{1} z_{1}^{-N}+\left(1-\rho_{0}-\rho_{1}\right) \delta_{N, 0}, \\
& Z_{N}^{\mathrm{BB}}=V\left(z_{0}\right) \rho_{0} z_{0}^{-N}+V\left(z_{1}\right) \rho_{1} z_{1}^{-N},
\end{aligned}
$$

where

$$
\rho_{0,1}=\frac{\left(1-z_{0,1} u\right)\left(1-z_{0,1} w\right)}{2-z_{0,1}(u+w)} .
$$

In our previous notation $\rho \equiv \rho_{0}$. The parameters $\rho$ and $\theta$ are related to the average energy per unit and the average magnetic moment per unit by 


$$
\begin{aligned}
& \lim _{N \rightarrow \infty} \frac{1}{N}\langle E\rangle_{N}^{\mathrm{if}}=\varepsilon=-J(1-4 \rho)-H(2 \theta-1), \\
& \lim _{N \rightarrow \infty} \frac{1}{N}\left\langle\left(\sum_{l} l n_{l}-\sum_{m} m n_{m}\right)\right\rangle_{N}^{\mathrm{if}}=\mu=2 \theta-1 .
\end{aligned}
$$

The correlation functions (4.5) are simple exponentials, for example

$$
g_{\mathrm{AB}}(R)=1+\left(\rho_{1} / \rho_{0}\right)\left(z_{0} / z_{1}\right)^{R}+\delta_{R, 0}\left(1-\rho_{0}-\rho_{1}\right) / \rho_{0} .
$$

By some rather elaborate algebra one may verify the susceptibility theorem (4.20). With the aid of the above formulae one easily finds explicit expressions for the quantities considered in the previous sections.

6. Discussion. We have studied the statistics of the occurrence of clusters of similar units in binary linear lattices starting from the assumption that the relative weight of an $\mathrm{A}_{l}$ or $\mathrm{B}_{m}$ cluster is determined only by its size $l$ or $m$. Of particular interest are the expressions derived for the average cluster numbers and the variance of their fluctuations for chains of a fixed number of units $N$ in the limit of large $N$. It has been shown that the statistics of clusters along the chain in this limit is determined completely by the cluster densities $\rho_{l}$ and $\rho_{m}$, and a correlation function $g_{\mathbf{A B}}(R)$. The susceptibility theorem (4.20) relates the integral of the correlation function to a thermodynamic derivative. In a forthcoming article ${ }^{12}$ ) we shall apply these methods and results to a theory of kinetics of binary linear systems.

\section{REFERENCES}

1) Poland, D. and Scheraga, H. A., Theory of Helix-Coil Transitions in Biopolymers, Academic Press (New York, 1970); this book also contains reprints of relevant papers, including refs. 2-10.

2) Hill, T. L., J. chem. Phys. 30 (1959) 383.

3) Zimm, B. H. and Bragg, J. K., J. chem. Phys. 31 (1959) 526.

4) Lifson, S. and Roig, A., J. chem. Phys. 34 (1961) 1963.

5) Lifson, S. and Zimm, B. H., Biopolymers 1 (1963) 15.

6) Lifson, S., J. chem. Phys. 40 (1964) 3705.

7) Litan, A. and Lifson, S., J. chem. Phys. 42 (1965) 2528.

8) Gibbs, J. H. and DiMarzio, E. A., J. chem. Phys. 30 (1959) 271.

9) Poland, D. and Scheraga, H. A., J. chem. Phys. 45 (1966) 1456; 45 (1966) 1464.

10) Fisher, M. E., J. chem. Phys. 45 (1966) 1469.

11) Felderhof, B. U., Ann. Phys. 58 (1970) 281.

12) Felderhof, B. U., to be published. 